\title{
Kritische Bemerkungen zur Bestimmung der pleistozänen Inlandeismächtigkeit mit Hilfe von Drucksetzungsmessungen
}

\author{
Von Günter VIEte, Freiberg/Sa. ${ }^{1}$ ) \\ Mit 1 Abbildung im Text
}

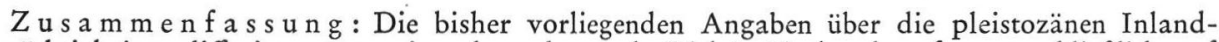
eismächtigkeiten differieren untereinander sehr stark (Tab.). Sie beruhen fast ausschließlich auf mehr oder weniger groben Abschätzungen und Vergleichen mit den rezenten Inlandcisgebieten. Es erscheint grundsätzlich möglich, zu genaueren Werten über die ehemaligen maximalen Eismächtigkeiten zu kommen, indem man mittels bodenmechanischer Messungen die durch die Eisbelastung in veränderlich - festen Lockergesteinen erfolgten Gefügeänderungen, d. h. die sogen. maximale Vorbelastung des Sedimentes bestimmt. Es werden die Ergebnisse von einigen an der interglazialen Kieselgur von Klieken bei Coswig, an glazigen gestörten und ungestörten Bändertonen von Niemegk (Fläming) und Sternberg (Mecklenburg), sowie versuchsweise an der Schreibkreide Rügens durchgeführten Messungen mitgeteilt und ihre geologische bzw. glaziologische Deutung diskutiert. Dabei ergeben sich durch die erforderlichen Annahmen über das Raumgewicht des z. T. verschutteten Eises und die Art und Mächtigkeit der zwischen Probeschicht und Eissohle liegenden Ablagerungen sowie durch die Schubbeanspruchung des Untergrundes seitens des sich bewegenden Eises einige z. T. erhebliche Fehlerquellen. Eine grundsätzliche Schwierigkeit stellt außerdem die Möglichkeit nachträglicher Gefügeänderungen, vor allem durch die kaltzeitliche Bodengefrornis, dar. Man wird daher auch durch derartige bodenphysikalische Untersuchungen nur in seltenen Einzelfällen zu genaueren Angaben über die pleistozänen Eismächtigkeiten kommen. Das zeigen auch die an mehreren Braunkohlenvorkommen Mitteldeutschlands durchgeführten Messungen der maximalen Vorbelastung (Tab.), von denen einige Ergebnisse zitiert werden.

S u m mary. Earlier informations about the thickness of Pleistocene inland ice always are based upon smaler or greater estimations and upon comparisons with recent inland ice. It seems possible to get better values of the former greatest thickness of ice by soil mechanical measurements of the so-called max. preliminary load of sediments with changeable solidness, ice-covered during the Pleistocene. The results of such measurements made on interglacial diatomaceous earth of Klieken near Coswig, on varved clay of Niemegk (Fläming) and Sternberg (Mecklenburg) and as an experiment on the chalk of Rügen are communicated. Their geological interpretation however is very difficult. Some partly considerable sources of errors result from the necessary assumptions about the weight of the ice, the kind and thickness of the layers embedded between the bottom of the ice and the trial bed. Further difficulties are the co-operation of shearing stress on the subsoil caused by the moving ice and the possibility of texture changes especially by freezing of the sediments during a cold period after its ice-covering. Therefore the determination of the thickness of Pleistocene inland ice by investigations based on soil physics will give accurate informations only in a few cases.

Bei verschiedenen glazialgeologischen Problemen - z. B. bei der Klärung der mechanischen Vorgänge am Boden einer sich bewegenden Eismasse oder bei der Abschätzung der Temperaturverhältnisse in den tieferen Teilen eines Inlandeises sowie in dessen Untergrund - spielt die jeweils vorhandene Eismächtigkeit eine wesentliche Rolle. Während aus den nivalen Gebieten der Gegenwart derartige Angaben mit Hilfe der angewandten Seismik relativ leicht zu erlangen sind, ist man bei der Ermittlung der pleistozänen Eismächtigkeiten fast nur auf mehr oder weniger hypothetische Abschätzungen angewiesen. Dementsprechend differieren die in der Literatur angegebenen Werte über die Dicke des pleistozänen Inlandeises in Nord- und Mitteldeutschland z. T. recht stark. Einige Beispiele mögen das erläutern (Tab. 1).

Grundsätzlich muß zunächst unterschieden werden zwischen Angaben über die Randdicke des Inlandeises während einer Vereisung in einem Gebiet und den Werten

1) Nach einem auf der 8. Hauptversammlung der DEUQUA am 23. 9. 1956 in Marburg/Lahn gehaltenen Vortrag; ergänzt und erweitert. 
Tabelle 1

Pleistozäne Inlandeisdicke in Mitteleuropa

\begin{tabular}{|c|c|c|c|}
\hline Autor & Gebiet & Eisdicke & Bemerkungen \\
\hline HeLland (1879) & $\begin{array}{l}\text { Berlin } \\
\text { mittlere Ostsee }\end{array}$ & $\begin{array}{l}370 \mathrm{~m} \\
650-700 \mathrm{~m}\end{array}$ & \\
\hline Dathe (1894) & mittlere Ostsee & maximal $4000 \mathrm{~m}$ & $\begin{array}{l}\text { Gefälle der Eisoberfläche mit } \\
1: 100 \text { angesetzt }\end{array}$ \\
\hline FRECH (1901) & $\begin{array}{l}\text { Trebnitzer } \\
\text { Katzengebirge }\end{array}$ & $\begin{array}{ll}\text { anfangs } & 100-200 \mathrm{~m} \\
\text { maximal } & 600-800 \mathrm{~m}\end{array}$ & \\
\hline ZiMMERMANN (1905) & Stettin & $3300 \mathrm{~m}$ & $\begin{array}{l}\text { Gefälle der Eisoberfläche mit } \\
0,5 \% \text { angesetzt }\end{array}$ \\
\hline Deecke (1906) & Stettin & $\begin{array}{l}1000 \mathrm{~m} \\
400-500 \mathrm{~m}\end{array}$ & $\begin{array}{l}\text { während der Hauptvereisung; } \\
\text { bei letzter Vereisung }\end{array}$ \\
\hline GraHMaNN (1925) & $\begin{array}{l}\text { Leipzig } \\
\text { Dresden }\end{array}$ & $\begin{array}{l}400 \mathrm{~m} \\
200 \mathrm{~m}\end{array}$ & $\begin{array}{l}\text { für die Elstereiszeit, Gefälle } \\
1: 500 \text { angesetzt }\end{array}$ \\
\hline PenCK (1933) & südl. Ostsee & $1000-1500 \mathrm{~m}$ & \\
\hline Bettenstaedt (1934) & Halle & $200-250 \mathrm{~m}$ & Hauptvorstoß der Saaleeiszeit \\
\hline GRIPP (1947) & Holstein & $100-130 \mathrm{~m}$ & für die letzte Eiszeit \\
\hline WAGENBRETH $(1955)$ & Profen b. Zeitz & $60 \mathrm{~m}$ & $\begin{array}{l}\text { für Saaleeiszeit; } \\
\text { Gefälle } 1: 1000 \text { angesetzt. }\end{array}$ \\
\hline
\end{tabular}

für die jeweiligen maximalen Eismächtigkeiten. Im erstgenannten Fall ist man fast ausschließlich auf Vergleiche mit den Verhältnissen an rezenten Eiskappen bzw. ihren Randgletschern angewiesen und muß dabei aber auch unbedingt die Gefällsverhältnisse der Eisoberfläche und den Bewegungszustand des Eises (Unterschiede zwischen Vorstoßund Rückzugsstadium!) berücksichtigen. Nur ausnahmsweise, wenn z. B. wie am Klingberg bei Sülfeld in Holstein junge Schliffspuren an einem in unmittelbarer Nähe des äußersten Eisrandes gelegenen Nunatak feststellbar sind (ILLIES), werden genauere direkte Angaben über randliche Eismächtigkeiten möglich sein. Die Abschätzung der maximalen Eisdicke in einem randfernen Gebiet ist praktisch nur mit der von GrahmanN (1925) angegebenen Methode einer Dreiecksberechnung möglich, d. h. Berechnung der Eismächtigkeit aus der Entfernung des Untersuchungsgebietes von der äußersten Randlage der betreffenden Vereisung unter der Annahme bestimmter Werte für die Randdicke und Oberflächenneigung des Eises sowie für die Gefälls- bzw. Geländeverhältnisse des Untergrundes. Während man über die beiden erstgenannten Faktoren auf Grund von Vergleichen mit den Verhältnissen in rezenten Inlandeisgebieten noch zu hinlänglich zuverlässigen Werten kommen kann, stellt die Abschätzung des ehemaligen Bodenreliefs vor allem für die älteren Vereisungen eine erhebliche Fehlerquelle dar. Man wird daher mit dieser Methode zwar für randnahe Gebiete zu brauchbaren Werten kommen, aber bei Abschätzungen z. B. der maximalen Dicke des elsterglazialzeitlichen Inlandeises im Gebiet der südlichen Ostsee nur sehr hypothetische Angaben erhalten. Insgesamt ergibt sich, daß alle diese Eisdickenangaben an gewisse Annahmen gebunden sind, und das Bestreben, auf irgendeinem Wege zu exakteren Werten zu kommen, ist daher durchaus verständlich.

Im ingenieurgeologischen Schriftum findet man nun in neuerer Zeit wiederholt Hinweise, daß nach den Arbeiten von Terzaghi und Casagrande durch Drucksetzungsmessungen an veränderlichfesten Lockergesteinen (vor allem Tonen) deren maximale Vorbelastung, d. h. auch eine frühere stärkere Belastung durch überlagernde Schichten und bei einem im Pleistozän eisbelasteten Sediment dessen maximale Eisbelastung, bestimmt werden kann. Damit ist also offenbar die Möglichkeit einer exakten Eisdickenbestimmung auf Grund von theoretisch wohl begründeten bodenphysikalischen Meß- 
werten gegeben. DüCKER (1951) hat entsprechende Versuche im Hinblick auf die Ermittlung der pleistozänen Inlandeismächtigkeiten in Nordeutschland angestellt und einige Ergebnisse mitgeteilt.

Die theoretischen Grundlagen für die Drucksetzungsmessungen und ihre Anwendung zur Bestimmung der maximalen Vorbelastung sind in jedem modernen Lehrbuch der Ingenieurgeologie zu finden. Das gesamte Verfahren ist relativ einfach. Zunächst werden (möglichst von Tonen oder tonigen Sedimenten) ungestörte Bodenproben entnommen und zwecks Vermeidung eines Wasserverlustes sofort einparaffiniert. Im Laboratorium werden aus diesen Proben Probekörper bestimmter Dimension vorsichtig ausgestochen, in einem Oddometer oder K-D- (Kompression - Durchlässigkeit-) Gerät stufenweise unter Druck gesetzt und die jeweiligen Setzungsbeträge gemessen. Aus der Belastungs-Setzungs-Kurve (auf log-Papier) kann dann auf einfache Weise die max. Vorlast bestimmt werden. Eine eingehende, sehr klare Darstellung des Meßverfahrens hat DüCKER in der genannten Arbeit gegeben.

Es bestehen kaum Zweifel, daß dieses Meßverfahren durchaus zuverlässig ist und zu exakten Werten der max. Vorbelastung führt - sofern in dem Sediment keine Gefügeänderungen nach seiner größten Belastung stattgefunden haben. (Auf diesen Punkt ist später noch einzugehen.) Die ersten Schwierigkeiten treten jedoch schon bei der geologischen Deutung des ermittelten Belastungswertes auf. Bei einem ehemals eisbedeckten Sediment setzt sich die geologische Auflast aus a) der Eislast und b) dem Gewicht der $\mathrm{zwischen}$ dem Eis und der untersuchten Schicht liegenden Sedimente zusammen.

$\mathrm{Zu}$ a). Das Raumgewicht des Eises darf nicht (wie im ingenieurgeologischen Schrifttum vereinzelt geschehen) generell mit $0,8-0,9 \mathrm{~g} / \mathrm{cm}^{3}$ angesetzt werden, da der unterste Teil der pleistozänen Gletscher und Inlandeismassen sicher mehr oder weniger mächtig durch die eingefrorene Grundmoräne verschuttet war. KELLER (1954) gibt für gefrorenen Geschiebelehm ein Raumgewicht von $2,1 \mathrm{~g} / \mathrm{cm}^{3}$ an und rechnet bei den bekanntlich stark verschutteten Toteismassen mit einem mittleren Raumgewicht für Eis + eingefrorene Grundmoräne von 1,5 g/cm. Das erscheint jedoch nur für abschmelzendes, schuttreiches Toteis zulässig. Bei aktivem vorstoßendem Eis müßte man für eine genaue Berechnung die Mächtigkeit der bodennahen Schuttzone kennen. Hier ist man jedoch nur auf allgemeine Annahmen bzw. Vergleiche mit rezenten Gletschern angewiesen. Bei den erheblichen Raumgewichtsdifferenzen von 2,1 und $0,9 \mathrm{~g} / \mathrm{cm}^{3}$ können somit in Hinblick auf die Abschätzung der ehem. Eisdicke bereits erhebliche Fehler auftreten.

Zu b). Zur genauen Ermittlung des Gewichtes der „Zwischenschichten“ müßte ihre Art und Mächtigkeit bekannt sein. Das ist aber sicher nur sehr selten der Fall. Eine Abschätzung der nachträglichen fluviatilen oder (bzw. und) glaziären Erosionswirkungen und -beträge ist meist garnicht oder nur sehr hypothetisch möglich. Wie will man z. B. bei der Untersuchung einer Septarientonprobe aus dem mitteldeutschen Raum feststellen, welche Ablagerungen in welcher Mächtigkeit sie zur Zeit der maximalen Eisbelastung überlagerten? Die einzige Möglichkeit, diese Schwierigkeit zu vermindern, bestände darin, die Messungen an interglazialen Ablagerungen in Gebieten, die anschließend nur noch einmal vom Eise überfahren wurden, durchzuführen. In Hinblick auf die stratigraphischen Unsicherheiten und die Bindung des Verfahrens ausschließlich an veränderlich-feste Lockergesteine wären derartige genau bestimmbare Messungen nur in Sonderfällen durchführbar. - In diesem Zusammenhang sei erwähnt, daß selbst bei hinlänglich genauer Bestimmung aller Faktoren im allgemeinen keine Aussage über die Zeit der maximalen Eisdicke möglich ist, die innerhalb der verschiedenen Vereisungsperioden und Eisvorstöße sicher wesentlich durch das örtlich vorhandene präglaziale Relief beeinflußt wurde. 
Im Folgenden sollen nun die Ergebnisse einiger Drucksetzungsmessungen mitgeteilt und jeweils die geologische Deutung der ermittelten max. Vorbelastung diskutiert werden.

Die Drucksetzungsmessungen wurden im Laboratorium für Bodenmechanik des Institutes für Tagebaukunde an der Bergakademie durchgeführt. Herrn Dipl.ing. Neumans sei für seine Unterstützung herzlichst gedankt.

1. Kieselgur von Klieken bei Coswig

Die Proben wurden aus der untersten „schweren Gur" entnommen. Sie waren überlagert von etwa $4 \mathrm{~m}$ Kieselgur (Raumgewicht $1,3 \mathrm{~g} / \mathrm{cm}^{3}$ ), 2,5 m tonigen Beckenablagerungen (etwa $1,6 \mathrm{~g} / \mathrm{cm}^{3}$ ) und $5-6 \mathrm{~m}$ Sanden (etwa $1,5 \mathrm{~g} / \mathrm{cm}^{3}$ ).

Maximale Vorbelastung: $5,8-6,0 \mathrm{~kg} / \mathrm{cm}^{2}$.

Deutung: Unter der Annahme, daß z. Zt. der Eisbedeckung zwischen der Probestelle und der Eisunterkante $4 \mathrm{~m}$ Gur und 2,5 m tonige Beckenablagerungen vorhanden waren und das Eis selbst eine etwa $10 \mathrm{~m}$ mächtige Zone mit Moränenschutt $\left(2,1 \mathrm{~g} / \mathrm{cm}^{3}\right)$ enthielt, ergibt sich eine Eisdicke (reines Eis mit eingefrorenem Grundmoränenschutt) von etwa $40-45 \mathrm{~m}$. Auch wenn man annimmt, daß die $Z_{\text {wischen- }}$ schichten noch mächtiger waren (z. B. entsprechend den heutigen Verhältnissen etwa $8-9 \mathrm{~m}$ ), bleibt immer noch eine zusätzliche Belastung, die einer rund $30 \mathrm{~m}$ mächtigen Eismasse entsprechen würde.

Aus diesem Ergebnis folgt, daß das Kieselgurlager von Klieken nochmals vom Eise überfahren worden sein muß. Dieser Befund stimmt mit den zu beobachtenden geologischen Verhältnissen (Stauchungen) überein, obwohl eine echte Grundmoräne im Hangenden der Gur nicht vorhanden ist. -

Vergleichsweise sei erwähnt, daß DüCKen (1951) an der Kieselgur der Lüneburger Heide folgende max. Belastungen feststellte: Munster $9,5 \mathrm{~kg} / \mathrm{cm}^{2}$, Wiechel $4,7 \mathrm{~kg} / \mathrm{cm}^{2}$, Schwindebek $0,8 \mathrm{~kg} / \mathrm{cm}^{2}$. Von diesen drei Vorkommen sind die beiden erstgenannten auch unter Berücksichtigung der überlagernden Schichten usw. sicher nochmals vom Eise überfahren worden.

2. Bänderton, Ziegelei am Kirstenhof bei Niemegk

Es handelt sich hier um Staubeckentone, die spätestens vor dem Eis der Warthevereisung abgelagert, anschließend vom Eise überfahren und z. T. stark glazigen gestaucht worden sind (vgl. Keilhack 1903, Viete 1953). Die zu untersuchenden Proben wurden aus einem nur sehr schwach gestörten Teil des Vorkommens entnommen. Heutige Überdeckung am Ort der Probenahme: $2,75 \mathrm{~m}$ Ton $\left(1,8 \mathrm{~g} / \mathrm{cm}^{3}\right)$ und $7 \mathrm{~m}$ Schmelzwassersande.

a. Lage der Druck-Testflächen parallel zur Schichtung und etwa parallel zur heutigen Tonoberfläche: maximale Vorbelastung $7,4-7,5 \mathrm{~kg} / \mathrm{cm}^{2}$. Rechnet man mit einer ehemaligen "Zwischenschicht" von $3 \mathrm{~m}$ Ton und wiederum $10 \mathrm{~m}$ verschuttetem Eis, so ergibt sich eine maximale Eisdicke von $\mathrm{rd} .70 \mathrm{~m}$, die somit wohl auch für die glazigenen Lagerungsstörungen der Bändertone verantwortlich zu machen wäre.

b. Lage der Druck-Testflächen senkrecht zur Schichtung mit einer N-S-Orientierung: maximale Vorbelastung $5,3 \mathrm{~kg} / \mathrm{cm}^{2}$.

c. Lage der Druck-Testflächen senkrecht zur Schichtung mit einer O-W-Orientierung: maximale Vorbelastung $4,2 \mathrm{~kg} / \mathrm{cm}^{2}$.

Auf die Deutung der beiden letztgenannten Ergebnisse wird im Anschluß an die Besprechung der Messungen eingegangen.

3. Beckentone, Ziegelei Sternberg ( Mecklenburg)

Im unteren Teil des Stoßes dieser westlich Sternberg, nahe der Straße nach Brüel gelegenen Ziegelei steht ein dunkler, fetter Ton an, der stark gestauchte und ver- 
knetete Fetzen von Bändertonen enthält und dementsprechend offenbar durch Eisdruck stark gestört ist. Er wird überlagert von einem völlig ungestörten braunen Ton mit einer sehr gut ausgeprägten Grob- und Feinbänderung (Abb. 1).

a. Proben aus dem unteren grauen Ton, etwa $10-20 \mathrm{~cm}$ unter dessen Oberkante; Druck-Testflächen parallel der Tonoberkante: maximale Vorbelastung 10,5 bis $11,5 \mathrm{~kg} / \mathrm{cm}^{2}$.

Deutung: Unter der Annahme, daß z. Zt. der Eisbedeckung eine „Zwischenschicht" von rund $1 \mathrm{~m}$ Ton $\left(1,9 \mathrm{~kg} / \mathrm{cm}^{2}\right)$ vorhanden und von dem Eis selbst wiederum eine Bodenzone von $10 \mathrm{~m}$ verschuttet war, erhält man eine Überlagerung von etwa $100-110 \mathrm{~m}$ Eis.

b. Proben wie a), jedoch Druck-Testflächen senkrecht zur Tonoberkante etwa NO-SW orientiert: $\max$. Vorbelastung $6 \mathrm{~kg} / \mathrm{cm}^{2}$.

c. Proben wie b), jedoch Druck-Testflächen etwa NW-SO orientiert: max. Vorbelastung $2,5 \mathrm{~kg} / \mathrm{cm}^{2}$.

Proben aus dem oberen, ungestörten, braunen Ton, etwa $0,7 \mathrm{~m}$ über dem grauen Ton entnommen. Überlagerung: $r d .2,5 \mathrm{~m}$ gebänderter Ton und 0,4 m Boden; DruckTestflächen parallel zur Schichtung des Tones. Maximale Vorbelastung: $8 \mathrm{~kg} / \mathrm{cm}^{2}$. Deutung: In Hinblick auf die glazigen völlig ungestörte Lagerung des oberen Tones erscheint die maximale Vorbelastung außerordentlich groß, denn selbst wenn man annimmt, daß die Probeschicht von $10 \mathrm{~m}$ Ton überlagert gewesen wäre, dürfte nur eine Belastung von etwa $2 \mathrm{~kg} / \mathrm{cm}^{2}$ auftreten. Das Meßergebnis zwingt also zu der Annahme, daß auch dieser Beckenton nochmals von einer mindestens $30-40 \mathrm{~m}$

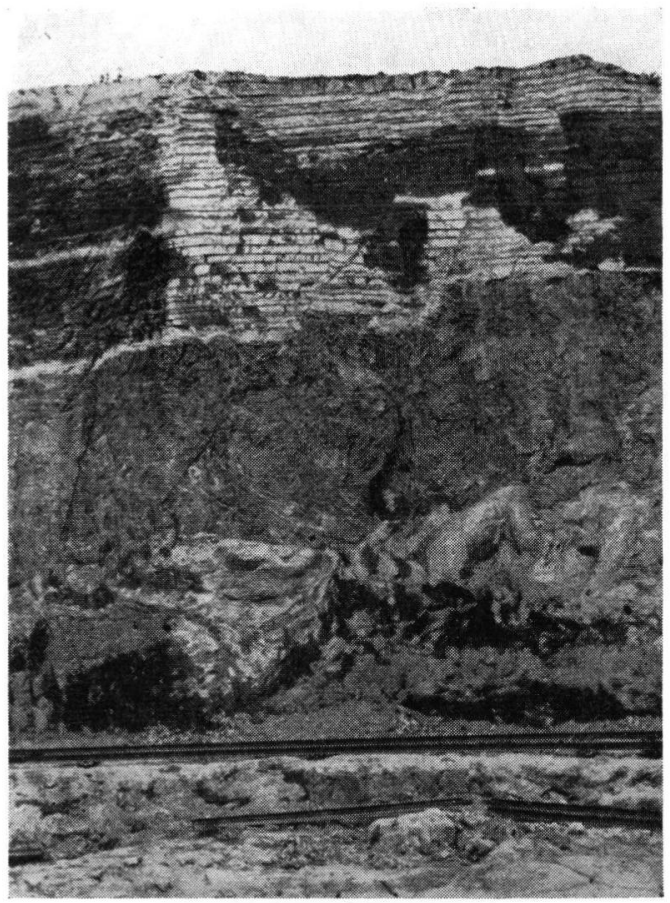

Abb. 1. Tongrube Ziegelei Sternberg (Mecklenburg). Im oberen Teil des Stoßes grob gebänderter, glazigen ungestörter Beckenton mit durch Sackungen (?) entstandenen Zerrsprüngen; darunter grauer, stark gestörter Beckenton mit zerrissenen und gefältelten, völlig unregelmäßigen Bändertonfetzen. Die Probenahme erfolgte etwa $20 \mathrm{~m}$ von diesem Ausschnitt nach links (westlich). 
mächtigen Eismasse überdeckt wurde. Die allgemeinen geologischen Verhältnisse sprechen jedoch eindeutig gegen eine derartige nochmalige Eisbedeckung, die sicher auch mehr oder weniger kräftige Lagerungsstörungen in den Tonen verursacht hätte. Die einzigen in dem oberen Beckenton vorhandenen Störungen sind einige scharf begrenzte Verwerfungen mit Sprunghöhen von einigen $10 \mathrm{~cm}$, wie sie in eiszeitlich gefrorenen Schichtpaketen - entstanden durch Setzungsvorgänge oder Sackungen im Untergrund infolge allmählichen Tieftauens - häufig zu beobachten sind. Es ergibt sich also hier eine deutliche Diskrepanz zwischen dem Meßergebnis und dem geologischen Befund, die zunächst nicht erklärbar ist.

4. Schreibkreide von Rügen

$\mathrm{Da}$ Versuche von Dr. JАСОВ, Freiberg/Sa., über die Zusammenhänge zwischen der Drucksetzung bzw. der maximalen Vorbelastung von Weichbraunkohlen und ihren Brikettiereigenschaften gezeigt haben, daß auch an Braunkohlen - d. h. Gesteinen, die nicht mehr zu den Lockerablagerungen i.e.S. gerechnet werden können - derartige Messungen erfolgreich durchgeführt werden können, wurde der Versuch unternommen, einige Proben der Rügener Schreibkreide auf ihre Vorbelastung zu untersuchen.

a. Probe aus dem alten Küster'schen Bruch bei Saßnitz mit einer heutigen Überlagerung von etwa $20 \mathrm{~m}$ Kreide und 3-4 m Geschiebemergel: max. Vorbelastung $20 \mathrm{~kg} / \mathrm{cm}^{2}$. Rechnet man mit einer früheren $Z$ wischenschicht von $25 \mathrm{~m}$ Kreide $\left(1,9 \mathrm{~g} / \mathrm{cm}^{3}\right)$ und $10 \mathrm{~m}$ verschuttetem Eis, so ergibt sich hier eine max. Eisdicke von $150-160 \mathrm{~m}$.

b. Probe wie a), jedoch Druck-Testfläche nicht horizontal, sondern senkrecht etwa in N-S-Richtung orientiert: max. Vorbelastung $13,5 \mathrm{~kg} / \mathrm{cm}^{2}$.

c. Probe wie b), jedoch Druck-Testfläche etwa O-W-orientiert: max. Vorlast $11,8 \mathrm{~kg} / \mathrm{cm}^{2}$.

d. Probe aus einem Kreidebruch bei Gummanz; bei einer heutigen Überlagerung von $\mathrm{rd} .10 \mathrm{~m}$ Kreide und $1 \mathrm{~m}$ Geschiebemergel: max. Vorbelastung 48,5 kg/ $\mathrm{cm}^{2}$. Setzt man in diesem Falle willkürlich eine ehemalige "Zwischenschicht" von $20 \mathrm{~m}$ Kreide sowie $10 \mathrm{~m}$ verschuttetem Eis an, so ergibt sich im Gegensatz zum Raum Saßnitz eine maximale Eismächtigkeit von etwa $480 \mathrm{~m}$ !

Obgleich bei der Deutung dieser Meßergebnisse über die Art und Mächtigkeit der während der größten pleistozänen Eisbedeckung vorhanden gewesenen Ablagerungen zwischen der Eisunterkante und der Probestelle infolge des Fehlens entsprechender geologischer Beobachtungen und wegen der Unklarheiten über die jungen tektonischen Verhältnisse bzw. Bewegungen in diesem Gebiet nur ganz willkürliche Annahmen gemacht werden können, bleibt der große Unterschied zwischen den Meßergebnissen von Saßnitz und Gummanz zunächst unerklärlich. Absolut genommen, erscheint die bei Saßnitz ermittelte Eisdicke von $150-160 \mathrm{~m}$ außerordentlich gering, denn allgemein nimmt man doch für die maximale pleistozäne Inlandeismächtigkeit (Elster- oder Saaleeiszeit) im Gebiet der südlichen Ostsee erheblich größere Werte an.

Betrachtet man die hier mitgeteilten Ergebnisse, so zeigt sich, daß einige mit den allgemeinen geologischen Befunden nur schwer oder garnicht in Einklang zu bringen sind. Außerdem muß festgestellt werden, daß die zur Deutung der maximalen Vorbelastung erforderlichen Annahmen über die Raumgewichtsverhältnisse des Inlandeises und die Mächtigkeit der "Zwischenschichten " mehr oder weniger große Unsicherheiten zur Folge haben und man die ermittelten Werte für die Eismächtigkeiten keineswegs als „exakt“ bezeichnen darf. Weitere Überlegungen über die Grundlagen der gesamten Methode zeigen nun, daß noch weitere Schwierigkeiten z. T. grundsätzlicher Art vorhanden sind. 
I. Bei der Deutung der maximalen Vorbelastung eines ehemals eisbedeckten Sedimentes ist zu berücksichtigen, daß sich die Druckbeanspruchung des Untergrundes durch eine bewegte Eismasse aus dem statischen Belastungsdruck und der $S \mathrm{chubk}$ raft des Eises zusammensetzt, wobei die letztere von der Bewegungsgeschwindigkeit des Eises abhängig ist. Bei der Zusammenpressung der Sedimente müssen somit streng genommen beide Komponenten berücksichtigt werden. Bisher ist es jedoch nicht möglich, genauere Aussagen über die Größe der Schubkraft und ihre Tiefenreichweite zu machen. Zweifellos dürfte ihr Anteil bei tiefer gelegenen Schichten zu vernachlässigen sein. Bei nur wenige Meter unter dem Eis gelegenen oder glazigen gestörten Sedimenten muß jedoch ihre Mitwirkung unbedingt berücksichtigt werden. Bei den vorstehenden Untersuchungen trifft das vor allem für die beiden Tonvorkommen von Sternberg und Niemegk zu. Da bei der Zusammenpressung des Sedimentes hier sicher eine Schubkomponente mitgewirkt hat, ergeben die nur eine statische Druckbeeinflussung berücksichtigenden Berechnungen zu große Eismächtigkeiten.

Um die Größenordnung dieser Schubkomponente wenigstens näherungsweise abschätzen zu können, wurden vom Niemegker Bänderton und vom unteren Sternberger Ton Proben ausgemessen, bei denen die Testflächen des Druckversuches senkrecht zur Schichtung bzw. Tonoberfläche und in verschiedenen Himmelsrichtungen orientiert waren. Die erhaltenen Werte setzen sich zusammen aus der allseitigen Zusammenpressung der Sedimente und ihrer glazigenen Schubbeanspruchung. Wenn man nun die Testflächen einmal genau in die Vorstoßrichtung des Eises und einmal senkrecht dazu orientiert was nur möglich ist, wenn nur eine Eisbedeckung des Sedimentes vorhanden war kann man beide Komponenten trennen und Hinweise über die Größe der Schubkraft erhalten. Leider ist es jedoch nur selten möglich, die durch das jeweilige präglaziale Relief bedingte lokale Vorstoßrichtung des Eises zu bestimmen.

Die Meßergebnisse von Niemegk (2 b, c) zeigen mit 5,3 bzw. 4,2 kg/ $/ \mathrm{cm}^{2}$, bezogen auf die Normalmessung von $7,4-7,5 \mathrm{~kg} / \mathrm{cm}^{2}$, relativ hohe Werte und geringe Differenzen untereinander. Es bestände jedoch die Möglichkeit, daß gerade hier, wo kräftige glazigene Lagerungsstörungen vorliegen, die Schubkomponente besonders groß war. Setzt man dieselbe einmal hypothetisch mit $3 \mathrm{~kg} / \mathrm{cm}^{2}$ an, so bliebe für die Eisdicke entsprechend ( $2 \mathrm{a})$ nur noch ein Wert von etwa $20 \mathrm{~m}$ übrig. - Bei dem unteren Sternberger Ton $(3 \mathrm{~b}, \mathrm{c})$ beträgt der Unterschied zwischen den NO- und NW-orientierten Messungen $3,5 \mathrm{~kg} / \mathrm{cm}^{2}$. Unter der Annahme, daß dieser Wert die Schubbeanspruchung darstellt, ergibt sich für die statische Belastung etwa $8 \mathrm{~kg} / \mathrm{cm}^{2}$ und gemäß (2 a) eine Eisdicke von rund $70-75 \mathrm{~m}$. - Es sei erwähnt, daß diese Angaben hier keineswegs als definitive Tatsachen gewertet werden, sondern nur die Schwierigkeiten und Fehlerquellen aufzeigen sollen, die mit der Eisdickenbestimmung nach der K-D-Methode verbunden sind.

II. Noch schwerwiegender als die bisher genannten Unsicherheiten erscheinen jedoch die folgenden grundsätzlichen Bedenken. Die gesamte Bestimmungsmethode beruht auf der Grundvoraussetzung, daß in den Ablagerungen nach ihrer maximalen Belastung keine Gefügeänderungen erfolgt sind. Derartige nachträgliche Veränderungen der Lage der Sedimentkörner zueinander können ganz allgemein z. B. durch stärkere Erschütterungen, Stoß- oder Druckwirkungen, $\mathrm{Zu}$ - oder Abnahme des Wassergehaltes sowie eine Gefrornis der Schichten verursacht worden sein.

a. Erschütterungen, Stoß-und Druckwirkungen durch natürliche Vorgänge sind in den hier zur Diskussion stehenden Gebieten und Ablagerungen im allgemeinen wohl zu vernachlässigen. Es werden zwar gelegentlich noch nachträgliche Sackungen und Setzungen von Schichtpaketen stattgefunden haben, aber die dabei auftretenden Gefügeänderungen sind sicher nur gering.

Zur Vervollständigung sei erwähnt, daß derartige Beeinflussungen auch im Zusammenhang mit tektonischen Vorgängen auftreten können. Obwohl eine ganze Reihe von Anzeichen 
für Schollenbewegungen in Nord- und Mitteldeutschland während des Pleistozäns vorliegen, ist es heute noch nicht möglich, genauere Angaben über diese z. T. auch jungquartäre Bruchtektonik zu machen. Es muß jedoch damit gerechnet werden, daß durch derartige Vorgänge vor oder nach der maximalen Eisbedeckung mehr oder weniger lokal Beeinflussungen des Korngefüges von Lockerablagerungen stattgefunden haben.

Recht wesentlich erscheinen ferner gewisse künstliche Beeinflussungen dieser Art, z. B. bei der Gewinnung von Probematerial durch Kernbohrungen oder bei der $\mathrm{Zu}-$ richtung der Probekörper für den Belastungsversuch (Einpressen eines Metallringes von $5-10 \mathrm{~cm}$ Durchmesser und $2 \mathrm{~cm}$ Höhe in die Bodenprobe oder bei Braunkohlen nach Neumann \& Jacob 1956 - Ausfräsen eines entsprechend großen Probekörpers mit einem Kernbohrer). Es besteht durchaus die Möglichkeit, daß durch derartige Bearbeitungen das Korngefüge gestört und damit das Meßergebnis beeinflußt wird.

b. Veränderungen des W a s e rgehaltes der Ablagerungen haben sicher mehrfach stattgefunden. Genauere Untersuchungen über die Beeinflussung des Wertes der maximalen Vorbelastung durch Feuchtigkeitsschwankungen stehen noch aus. Vorläufige Vergleichsmessungen an Proben, die vom luftrockenen Stoß entnommen worden waren, ergaben Differenzen gegen "normal“ bergfeuchte Proben von $0,5-1,5 \mathrm{~kg} / \mathrm{cm}^{2}$. Diese Werte sind relativ gering, können aber gelegentlich von ausschlaggebender Bedeutung sein. Unbekannt ist vor allem auch das Verhalten des Korngefüges bei einem mehrmaligen Wechsel von stärkerer und geringerer Durchfeuchtung bzw. Austrocknung. Mit dieser Beeinflussung hängen eng die Gefügeänderungen infolge

c. Gefrornis und A uf ta u en der belasteten Sedimente zusammen. Bekanntlich sind bei der Bodengefrornis verschiedene Bodenfroststrukturen - wie kompaktes Bodeneis, Eislinsen, Eisschichten usw. - zu unterscheiden. Bei ihrer, u. a. von Bodenart, Korngrößenverteilung, Frostintensität und -eindringungsgeschwindigkeit abhängigen Entstehung findet teils eine Wasserauspressung, bei feinkörnigen Ablagerungen jedoch meist eine Wasseranreicherung aus der Umgebung statt. Dabei erfolgen in den Lockerablagerungen Veränderungen des Korngefüges und zwar vor allem Gefügelockerungen, deren Stärke von der Art, Schnelligkeit und Dauer der Gefrornis, den Korngrößenverhältnissen, der Wasseranreicherung usw. bestimmt wird. Besonders starke Lockerung erfolgt in Bereichen großer Eisanreicherung sowie in Eislinsen, -adern und -schichten, während in deren Umgebung durch Wasserentzug möglicherweise umgekehrt eine Kornverdichtung stattfinden kann. Beim Auftauen einer gefrorenen Lockerablagerung wird die Gefügelockerung mit der allmählichen Wasserabgabe zurückgehen, aber es wird sicher ein gewisser irreversibler Lockerungsrest erhalten bleiben - sofern nicht infolge starker Wasserübersättigung Kornumlagerungen erfolgten, wodurch das ehemalige Korngefüge dann selbstverständlich restlos zerstört wird. - In Hinblick auf die hier zur Diskussion stehenden Fragen ergibt sich zunächst, daß man Proben für Drucksetzungsmessungen nur in den frostfreien Jahreszeiten und zwar aus den Teilen eines Schichtenpaketes entnehmen darf, die außerhalb des Bereiches der winterlichen Gefrornis liegen. Neben dieser winterlichen muß aber auch die e is zeitliche Bodengef rornis berücksichtigt werden. Es bestehen wohl heute keine Zweifel mehr darüber, daß während der pleistozänen Kaltzeiten im gesamten mitteleuropäischen Periglazialgebiet ein mehr oder weniger tiefgründiger Dauerfrostboden mit einer nur wenige Dezimeter bis Meter mächtigen sommerlichen Auftauzone vorhanden war. Die Tiefenreichweite dieser Gefrornis wird man unter Berücksichtigung der kaltzeitlichen Klimaverhältnisse und der Situation in den heutigen subpolaren Tundren- bzw. Peri-glazialgebieten mit mindestens einigen zehn Metern (zunehmend von Westen nach Osten) ansetzen können. Ein erheblicher Teil der pleistozänen und z. T. auch der präquartären Sedimente im mitteldeutschen Raum muß somit infolge dieser Gefrornis und des Wechsels der Kaltzeiten und Interstadial- bzw. Interglazialperioden Gefügeänderungen erlitten haben. Eine Abschätzung über die Stärke der Beeinflussung des Wertes der maxi- 
malen Vorbelastung durch diese Veränderungen des Korngefüges dürfte kaum möglich sein, da die hier mitwirkenden Variabeln, wie Art der Gefrornis, Wassergehalt des Sedimentes, Tiefe der Frosteinwirkung und des Auftauens, Häufigkeit des Wechsels zwischen Gefrornis und völligem Auftauen usw., wohl niemals sicher ermittelt bzw. rekonstruiert werden können. Zudem liegen bisher auch noch keine experimentellen Untersuchungen zu diesem Fragenkomplex vor. Drei Proben, die in gefrorenem Zustand entnommen und langsam aufgetaut wurden, ergaben in zwei Fällen um 2 bzw. $3,5 \mathrm{~kg} / \mathrm{cm}^{2} \mathrm{zu}$ geringe, in einem um $2,4 \mathrm{~kg} / \mathrm{cm}^{2} \mathrm{zu}$ hohe Werte. Abschließend ist somit festzustellen, daß diese Gefügeänderungen den Wert der gesamten Methode für die Eisdickenbestimmung sehr stark vermindern. Man wird daher die im Vorangegangenen abgeleiteten Zahlen zwar als schätzungsweise mögliche, jedoch keineswegs sichere Angaben ansehen dürfen.

Uberblickt man diese Erörterungen, so ergibt sich folgendes:

1. Die K-D-Methode führt entsprechend ihrer theoretischen Grundlagen zu exakten Werten für die maximale Vorbelastung eines veränderlich-festen Lockergesteins, sofern in diesem keine sekundären Veränderungen des Gefüges stattgefunden haben.

2. Bei der geologischen Deutung der maximalen Vorbelastung ergeben sich durch die Unkenntnis eventueller früherer Erosionswirkungen, der Zusammensetzung und Mächtigkeit der ehemaligen hangenden Schichtenfolge, tektonischer Beanspruchungen usw. mehrere Unsicherheitsfaktoren.

3. Die gleichen Faktoren treten bei der Ermittlung der maximalen pleistozänen Inlandeismächtigkeit aus der Vorbelastung ehemals eisbedeckter Sedimente auf. Dazu gesellen sich als weitere Schwierigkeiten: Abschätzung der Raumgewichtsverhältnisse des z. T. verschutteten Inlandeises, Mitwirkung einer Schubkomponente bei der Zusammenpressung der Ablagerungen des Untergrundes und nachträgliche Gefügeänderungen durch die eiszeitliche Bodengefrornis.

Damit kann festgestellt werden, daß aus der maximalen Vorbelastung zwar gewisse Hinweise über die ehemalige Inlandeismächtigkeit abgeleitet werden können, aber in keiner Weise als exakt zu bezeichnende und auch kaum zuverlässigere Werte zu erhalten sind, als z. B. nach den erwähnten Abschätzungen mit der Dreiecksmethode.

Ergänzend sei noch auf einige bei der Bestimmung der maximalen Vorbelastung von Weichbraunkohlen erzielten Ergebnisse hingewiesen (vgl. NeumanN \& JАСОв 1956).

Tabelle 2

Maximale Vorbelastung einiger Braunkohlenflöze (n. Neumann \& Jасов 1956)

\begin{tabular}{|c|c|c|c|c|}
\hline Gebiet & Ort & geol. Alter & \multicolumn{2}{|c|}{ max. Vorbelastung } \\
\hline Lausitz & Piskowitz & Unter-Miozän & \multicolumn{2}{|c|}{$8-11 \mathrm{~kg} / \mathrm{cm}^{2}$} \\
\hline Oberlausitz & Berzdorf & ” & $22-25$ & $"$ \\
\hline \multirow{3}{*}{$\begin{array}{l}\text { Niederlausitz } \\
\text { (Unterflöz) }\end{array}$} & Kleinleipisch & ” & $21-30$ & 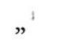 \\
\hline & Niemtsch & ” & $24-30$ & ” \\
\hline & Klettwitz & ” & $27-31$ & ” \\
\hline $\begin{array}{l}\text { Borna } \\
\text { (Hauptflöz) }\end{array}$ & Schleenhain & Unter-Oligozän & $32-43$ & ” \\
\hline Bitterfeld & Holzweissig & Ober-Oligozän & $39-64$ & ” \\
\hline Geiseltal & Kayna & Mittel-Eozän & $50-63$ & » \\
\hline Harzrand & $\begin{array}{l}\text { Nachterstedt } \\
\text { Oberflöz } \\
\text { Unterflöz }\end{array}$ & $”$ & $\begin{array}{l}70-79 \\
49-70\end{array}$ & $”$ \\
\hline
\end{tabular}


Nach einleitenden Vorversuchen über die Setzungsbeträge und Kompressibilität von Torfen und Braunkohlen, die Einflüsse des Wassergehaltes usw. haben die genannten Verff. jeweils mit mehreren Proben die maximale Vorbelastung einiger Braunkohlenflöze bestimmt (Tab. 2). Wollte man aus diesen Zahlen die jeweilige maximale Inlandeismächtigkeit bestimmen, so würden sich auch unter Berücksichtigung der Deckgebirgsmächtigkeiten recht eigenartige Verhältnisse ergeben. Es zeigt sich allgemein, daß die max. Vorbelastung und auch die Schwankungsbreite der Meßergebnisse mit zunehmendem Alter der Kohlen zunimmt. Der Unterschied zwischen den etwa gleichaltrigen Kohlen von Piskowitz und Berzdorf könnte mit den starken glazigenen Lagerungsstörungen der letzteren in Verbindung gebracht werden - aber die übrigen weniger stark gestörten Kohlen der Niederlausitz zeigen gegenüber Berzdorf keine wesentlich anderen Werte. Sehr auffällig ist außerdem, daß das Nachterstedter Oberflöz eine größere max. Vorbelastung aufweist als das Unterflöz! Durch Setzungsversuche an verschiedenen Torfen konnten die Verff. nun nachweisen, daß die Kompressibilität derartiger Ablagerungen wesentlich von der Art der beteiligten pflanzlichen Substanzen, d. h. von der Fazies abhängig ist. Das muß sich selbstverständlich bei den durch Drucksetzungsmessungen ermittelten Werten der maximalen Vorbelastung von faziell verschiedenen Braunkohlen ebenfalls bemerkbar machen. Auch regionale Unter$\mathrm{schiede}$ des Inkohlungsgrades als Funktion von Fazies, Alter und geologischer Geschichte wirken sich aus. Somit ist auch hier festzustellen, daß die geologische Deutung der gemessenen max. Vorlasten recht schwierig und problematisch ist. Daher wird man den oben angegebenen Messungen an der Schreibkreide Rügens ebenfalls keine wesentliche Aussageberechtigung beimessen dürfen.

Schließlich muß erwähnt werden, daß nach Versuchen von NeumanN \& JACOB Torfe und Tone bei Belastungen sehr unterschiedliche Setzungsbeträge aufweisen. Daraus muß geschlossen werden, daß die Drucksetzungskurven verschiedener Sedimente nicht direkt vergleichbar sind. Damit wird die Anwendungsmöglichkeit der K-D-Methode zur Erlangung von Aussagen über die geologische Vorbelastung weiterhin eingeschränkt.

\section{Literatur}

DüCKER, A.: Ein Untersuchungsverfahren zur Bestimmung der Mächtigkeit des diluvialen Inlandeises. - Mitt. geol. Staatsinst. Hamburg 20, S. 3, 1951.

Grahmann, R.: Diluvium und Pliozän in Nordwestsachsen. - Abh. sächs. Akad. d. Wiss., Math.phys. K1. 39, Nr. 4. 1925.

KeILHACK, K.: Geologische Beobachtungen während des Baues der Brandenburgischen Städtebahn. - Jb. preuß. geol. Landesanst. 24, S. 1, Berlin 1903.

Keller, G.: Drucktexturen in eiszeitlichen Sedimenten. - Eiszeitalter und Gegenwart 4/5, S. $158,1954$.

Neumann, K. \& Jасов, H.: Drucksetzungsversuche mit Weichbraunkohlen. - Z. f. angew. Geologie 2, S. 307, Berlin 1956.

VIETE, G.: Über zwei quartärgeologische Beobachtungen bei Niemegk (Fläming). - Geologie 2, S. 131, Berlin 1953.

Manuskr. eingeg. 28. 11. 1956.

Anschrift des Verf.: Dr. Günter Viete, Geologisches Institut der Bergakademie Freiberg, Freiberg/Sachsen. 